\title{
Le commentaire de Josse Bade aux comédies de Térence
}

\author{
Laure Hermand-Schebat
}

\section{(2) OpenEdition}

1 Journals

Édition électronique

URL : http://journals.openedition.org/rhetorique/562

DOI : $10.4000 /$ rhetorique.562

ISSN : 2270-6909

Éditeur

UGA Éditions/Université Grenoble Alpes

Édition imprimée

ISBN : 978-2-37747-029-7

Référence électronique

Laure Hermand-Schebat, "Le commentaire de Josse Bade aux comédies de Térence », Exercices de rhétorique [En ligne], 10 | 2017, mis en ligne le 26 décembre 2017, consulté le 02 octobre 2020. URL : http://journals.openedition.org/rhetorique/562 ; DOI : https://doi.org/10.4000/rhetorique.562

Ce document a été généré automatiquement le 2 octobre 2020.

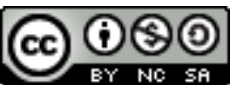

Les contenus de la revue Exercices de rhétorique sont mis à disposition selon les termes de la Licence Creative Commons Attribution - Pas d'Utilisation Commerciale - Partage dans les Mêmes Conditions 4.0 International. 


\title{
Le commentaire de Josse Bade aux comédies de Térence
}

\author{
Laure Hermand-Schebat
}

1 "Pour le lecteur du XvI e siècle ", comme l'affirme Harold Walter Lawton, «les noms de Térence et Donat étaient comme inséparables ${ }^{1} »$. Si l'on dénombre plus de cinq cents éditions des comédies de Térence de 1470 à 1600 , elles sont souvent accompagnées du commentaire de Donat, sauf pour l'Heautontimoroumenos dont le commentaire manque dans les manuscrits ${ }^{2}$, et elles comportent plus fréquemment encore, en ouverture, le $D e$ fabula (ou De tragoedia et comoedia) d'Évanthius, attribué à l'époque lui aussi à Donat. Les commentateurs de Térence à la Renaissance ont donc à leur disposition à la fois un modèle et une source d'informations. Les philologues les plus érudits utilisent ce commentaire comme une source parmi d'autres sur le théâtre antique; c'est le cas de l'humaniste florentin du Quattrocento, Ange Politien, dont le commentaire aux deux cents premiers vers de L'Andrienne révèle entre autres une connaissance riche et précise des sources grecques du théâtre antique ${ }^{3}$. Mais pour les humanistes qui connaissent peu ou pas le grec, comme c'est le cas de l'imprimeur parisien de la première moitié $\mathrm{du} \mathrm{xvI}^{\mathrm{e}}$ siècle, Josse Bade, Donat reste la référence et la source principale concernant le théâtre antique. Il fait en effet paraître en 1502 son commentaire de Térence : il s'agit d'ajouts (une longue préface intitulée Praenotamenta et des sections de commentaire de L'Andrienne et de L'Eunuque) qui prennent place au sein d'une édition proposant le commentaire de Guy Jouenneaux que Bade avait déjà publié chez Trechsel en $1493^{4}$. Ce commentaire s'appuie largement sur celui de Donat.

2 Ce que puise Bade chez Donat, c'est d'abord une explication linéaire du texte térentien. Dans sa préface datée de 1501, adressée au jurisconsulte Hervé Besin, Josse Bade désigne son ouvrage par l'expression «nos explications de Térence» («nostra in Terentium elucidamenta »). Il souligne ensuite, même si cet élément est un topos du genre de la lettre dédicatoire, la modestie et la simplicité de son commentaire :

Sunt etenim sermone fere plebeio atque humili, eruditionis, ni fallor, bone atque sententiarum utilium plus quam salis atque elegantiarum habentia; quippe uulgo, ut diximus, illiterato destinata. 
Elles [nos explications] sont en effet écrites dans un langage courant et simple et sont pourvues, si je ne m'abuse, d'un honnête savoir et de maximes utiles plus que d'esprit et de raffinement ; c'est pourquoi elles sont destinées, comme je l'ai dit, à un large public non lettré.

Le commentaire de Josse Bade ne se présente donc pas comme un commentaire érudit, mais comme un commentaire de vulgarisation. L'aspect technique, plutôt complexe, des figures de rhétorique ${ }^{5}$ y est par exemple laissé de côté. S'appuyant sur le De fabula d'Évanthius, Bade cherche également à transmettre à son lecteur une somme d'informations sur le théâtre antique et les conditions matérielles de sa représentation. Comme nous le verrons, sont consacrés au théâtre antique plusieurs chapitres des Praenotamenta, sorte de longue introduction confinant au traité de poétique en miniature, que Bade place en introduction à son commentaire. Ce sont d'ailleurs davantage les questions de poétique que de technique rhétorique qui intéressent Bade, et sa réflexion se nourrit à ce sujet tout autant du commentaire de Donat que d'autres sources telles que Cicéron, Quintilien ou les grammairiens de l'Antiquité tardive.

\section{Un imprimeur humaniste}

3 Josse Bade (Jodocus Badius Ascensius en latin) est l'un des imprimeurs les plus importants de la première moitié $\mathrm{du} \mathrm{XVI}{ }^{\mathrm{e}}$ siècle. Ce sont 775 éditions, imprimées pour lui-même ou en association avec d'autres imprimeurs, qui sortent de ses presses entre 1503 et 1535, date de sa mort. Ses contacts avec les milieux humanistes font de lui « un imprimeur et un érudit » pour reprendre la formule de Paul White qui lui a consacré récemment une monographie ${ }^{6}$.

4 Après une jeunesse passée à Gand, il étudie vraisemblablement à Louvain, puis en Italie dans les années 1480 ; il séjourne à Ferrare, où il écoute les leçons de grec de Battista Guarino, et à Bologne, ville dans laquelle il suit les cours de Filippo Beroaldo l'Aîné. Il est à Lyon en 1492: il édite les Orationes de Beroaldo chez Jean Trechsel, imprimeur d'origine germanique installé à Lyon, qui fut l'un des premiers typographes de la ville. Il s'agit du premier des volumes parus chez cet imprimeur sous sa direction. Il collabore avec Trechsel de 1492 à 1499. Il enseigne à la même époque la littérature latine dans un collège lyonnais tenu par Henri Valluphin. Cette première expérience de l'enseignement est importante pour son activité d'imprimeur et même déterminante quant à son travail de commentateur des textes classiques, patristiques et humanistes. Comme nous le verrons, Bade construit son ethos humaniste autour de cette activité, en complément de celle d'imprimeur. Il propose une vision pédagogique du métier d'imprimeur et bon nombre de ses éditions et de ses commentaires témoignent d'un réel et profond souci pédagogique.

5 Il arrive à Paris en 1499. Entre 1499 et 1502, il prépare des éditions pour divers imprimeurs parisiens, financées par Jean Petit. Il établit sa propre presse en 1503 ; son atelier, le Praelum Ascensianum, était situé rue saint Jacques. Il fait paraître ses dernières publications en 1534. Sa carrière comme imprimeur s'étale donc sur une trentaine d'années d'activité. Parmi ses 775 éditions, 182 sont des éditions de textes de l'Antiquité profane, essentiellement latins. Il joue un rôle essentiel de transmission et de diffusion des textes antiques classiques au $\mathrm{xvI}^{\mathrm{e}}$ siècle. Il est également actif dans le domaine humaniste et contribue de manière décisive à la diffusion en France de l'humanisme italien (selon Louise Katz, $20 \%$ de sa production sont consacrés à l'édition d'œuvres d'humanistes transalpins). Il établit entre autres des éditions de Crinito, Politien, Valla 
et de son poète préféré, Battista Spagnuoli, dit le Mantouan. Il est également un des éditeurs attitrés d'Érasme, même si l'engagement de Bade contre le luthérianisme semble avoir scellé leur rupture définitive dont témoigne la vengeance d'Érasme en 1528 dans le Ciceronianus qui compare les mérites stylistiques de Josse Bade avec ceux de Guillaume Budé, ce qui discrédite l'imprimeur dans les milieux humanistes.

Josse Bade se forge une réputation d'érudit et de typographe habile. Il rédige une foule de paratextes (épîtres dédicatoires, préfaces, commentaires, poèmes) à ses nombreuses éditions d'auteurs antiques et renaissants. Ces paratextes font de lui un véritable «imprimeur-humaniste» pour reprendre l'expression de Louise Katz ${ }^{7}$. Comme le souligne Paul White, l'imprimeur parisien est en contact avec les cercles de Jacques Lefèvre d'Étaples et de Guillaume Budé, il fait appel aux compétences philologiques de Beatus Rhenanus et réunit autour de lui un groupe de jeunes érudits, les «Ascensiani »: François Du Bois, Pierre Danès et Jacques Toussain ${ }^{8}$. Mais il faut toutefois noter que ses éditions témoignent d'un intérêt mineur pour les questions textuelles et d'une préoccupation plus grande pour les aspects moraux et spirituels. Paul White y voit une tendance de l'humanisme en Europe du Nord par opposition à l'humanisme italien qui privilégie les questions philologiques. Il faut en tout cas souligner chez Bade la visée morale du travail philologique. Selon Louise Katz, «il place ainsi l'apprentissage des "bonnes lettres" et des "bonnes mœurs" au cœur de sa politique éditoriale dans la transmission tant des classiques de l'Antiquité profane que des œuvres de l'humanisme italien ${ }^{9}$ ».

\section{Un commentaire aux multiples éditions}

7 Dès ses années lyonnaises, Josse Bade témoigne d'un intérêt marqué pour les pièces de Térence. Pendant qu'il collabore dans les années 1490 avec l'imprimeur lyonnais Johannes Trechsel, Bade supervise une édition commentée des pièces de Térence. Elle paraît à Lyon en 1493 sous le titre Guidonis Iuuenalis natione Cenomani in Terentium familiarissima interpretatio cum figuris unicuique scaenae praepositis ${ }^{10}$. Cette édition a été étudiée et est fréquemment citée par les historiens du théâtre, car ses nombreuses gravures sur bois, 159 exactement, donnent des représentations de l'espace théâtral avant la scénographie italienne. Il s'agit d'une édition illustrée (cum figuris), mais aussi d'une édition commentée (interpretatio) : Bade ne rédige pas lui-même de commentaire, mais reprend un commentaire dû à Guy Jouenneaux (Guido Iuuenalis en latin), grammairien et théologien originaire du Mans, actif à la fin $d u x^{e}$ siècle ${ }^{11}$. Les paratextes de l'édition sont aussi de Guy Jouenneaux : deux lettres préliminaires, trois autres lettres et un poème à la fin de l'ouvrage, intitulé Guidonis Iuuenalis Natione Cenomani / epigramma, super causas operis suscepti, viennent compléter l'édition commentée elle-même. L'édition des comédies de Térence qui sort des presses de Trechsel en 1493, a été en fait placée sous la responsabilité éditoriale de Bade. C'est lui qui fait le travail d'édition du texte, il y ajoute le commentaire de Guy Jouenneaux ; et c'est probablement lui qui supervise l'illustration et en organise les gravures, même si ce n'est pas lui qui en effectue la réalisation.

8 Cette édition n'est pas d'ailleurs à proprement parler la première édition de Térence coordonnée par Bade. Il revient à Louise Katz d'avoir découvert une édition badienne, antérieure aux Orationes de Beroaldo publiées chez Trechsel en 1492: il s'agit d'« une édition des Comédies de Térence imprimée par Mathieu Husz, le 5 novembre 1491, et 
pour laquelle Bade compose une épître qu'il adresse à Laurent Bureau, son premier protecteur $^{12}$ ». Cette édition fournit le commentaire de Donat que Bade cite à plusieurs reprises dans son épitre dédicatoire. Bade travaille donc, dès 1491 au moins, sur le texte de Térence. Ce travail d'édition est probablement en lien avec son activité d'enseignant puisque dans les années 1490, il donne des cours de littérature latine dans un collège lyonnais tenu par Henri Valluphin, cours qui incluent certainement un commentaire du texte térentien, devenu un classique scolaire dès l'Antiquité tardive.

9 Le commentaire de 1502 dû à Bade est l'aboutissement de cette activité pédagogique : analysant les longues listes systématiques que contient le commentaire, Paul White en souligne la proximité avec les pratiques scolaires ${ }^{13}$. Ainsi, la section intitulée De doctrinis \& autoritatibus ex prologo notandis, insérée dans le commentaire du prologue de l' Andrienne, propose une liste de dix leçons morales à tirer de ce prologue. De même, le commentaire du premier acte contient une liste intitulée Principales sententiae et auctoritates primae scenae primi actus qui récapitule dix principes moraux principalement destinés à l'éducation des jeunes gens, Bade fournissant une méthode précise de lecture des sententiae. On peut voir dans ces listes numérotées la trace de leçons données par Bade à ses élèves, chargés de recopier et d'apprendre ce type de listes. Un certain nombre d'éléments du commentaire de Térence reflètent donc la pratique d'enseignant de Bade $^{14}$. De manière plus générale, ce commentaire, comme plusieurs autres dus à Bade, témoigne d'un grand souci pédagogique.

10 J'ai cru pendant un temps avoir trouvé confirmation de cette hypothèse dans une préface attribuée à Bade par Maurice Lebel ${ }^{15}$. Mais cette attribution s'est révélée erronée. Maurice Lebel reproduit en effet dans son ouvrage un texte liminaire à l'édition badienne des tragédies de Sénèque sortie de sa presse parisienne en $1514^{16}$. Il s'agit d'une lettre-préface adressée à Guillaume de Rochefort, grand chancelier de France, mentionnée par Philippe Renouard dans sa description de l'ouvrage ${ }^{17}$, mais dont le texte n'est pas reproduit. En effet, comme le mentionne clairement la formule d'adresse de la lettre, ce texte n'est pas dû à Bade, mais à Gellio Bernardino Marmitta, homme de lettres actif à Parme à la fin du $\mathrm{Xv}^{\mathrm{e}}$ siècle, qui a rédigé un commentaire aux tragédies de Sénèque publié à Lyon en 1491 par Antoine Lambillon et Marin Sarrazin et dont Bade a probablement eu connaissance pendant ses années lyonnaises. Il était tout à fait tentant d'attribuer à Bade les propos suivants :

Quod quidem mihi hoc tempore faciendum esse duxi, non spe aliqua emolumenti, sed amore et studio honestatis, ut si quid vigiliis aut bonarum artium disciplina ipse perfeci, id omnibus libenter impertiri velim. Itaque has commmentationes quas superioribus annis, dum publice profiterer, forte indigeste inconditeque annotavimus, te hortante nunc imprimendas dedimus.

Voilà ce que j'ai cru devoir faire à notre époque, non pas dans l'espoir de recevoir quelque émolument, mais par amour et par goût pour le bien, de sorte que tout ce que j'ai accompli par mes veilles et mon application à la bonne littérature, je veux de tout cœur que tous en bénéficient. C'est dans cet esprit que, sur votre recommandation, j'ai finalement envoyé à l'impression les commentaires que j'avais mis par écrit en vrac et sans apprêt, au petit bonheur, alors que je donnais des cours publics durant ces dernières années ${ }^{18}$.

Ces mots ne sont malheureusement pas de lui! Mais même si ce lien entre son enseignement et ses commentaires d'auteurs classiques n'est pas fait explicitement par Bade lui-même, l'hypothèse n'en demeure pas moins hautement probable.

11 La première édition du commentaire de Bade proprement dit, comprenant les Praenotamenta et les ajouts de Bade au commentaire de Jouenneaux, date, nous l'avons 
$\mathrm{vu}$, de $1502^{19}$. Elle ne comporte plus d'illustrations. La majeure partie du commentaire de Térence reprend toutefois l'édition de 1493 avec le commentaire de Jouenneaux. Les ajouts badiens sont concentrés sur les deux premières pièces de Térence, L'Andrienne et L'Eunuque. C'est le commentaire à L'Andrienne qui en contient le plus. Autre exception notable : le prologue de L'Eunuque qui recourt de nouveau à la méthode expérimentale de commentaire élargi, développée pour le début de L'Andrienne, dans des proportions moindres toutefois. Bade mentionne toujours ses interventions au fil du commentaire. Pour clore son commentaire à L'Andrienne I, 1 , il insère une section intitulée Principales sententiae et auctoritates primae scene primi actus et ajoute à la fin du titre la mention per Ascensium pour revendiquer la paternité de cette liste. En bas de la même page, il clôt sa section des « Notes préliminaires » par les deux phrases suivantes :

Finiuntur prenotamenta precipua Jodoci Badii Ascensii. Sequuntur familiares interpretationes Domini Guidonis Juvenalis.

Fin des notes préliminaires propres à Josse Bade. Viennent ensuite les explications simples de dom Guy Jouenneaux.

Quelques pages plus loin, à la fin du commentaire de la première scène de L'Andrienne par Guy Jouenneaux, l'ajout d'une note de Bade sur le vers 165 («Sine eveniat») est marqué par la mention Ascensius avant le passage à la scène suivante entre Simon et Dave. Le commentaire apparaît ainsi comme un texte comportant différentes strates ${ }^{20}$. C'est un genre qui garde indéniablement la trace de la tradition scolaire. Paul White considère cette édition de 1502 comme ce qu'il appelle « le coup d'essai » d'un nouveau type de commentaire ${ }^{21}$; mais il signale la distribution inégale $d u$ volume $d u$ commentaire et y voit le reflet de la réalité de la salle de classe.

Le commentaire de Josse Bade aux comédies de Térence, tel qu'il apparaît dans l'édition de 1502, connut de nombreuses rééditions, à commencer par celle de 1504 sur laquelle nous avons travaillé, publiée par Bade en collaboration avec des imprimeurs londoniens ${ }^{22}$. Comme le souligne Paul White, les notes de Bade sur Térence furent très populaires, car reprises une cinquantaine de fois dans des éditions du XvI ${ }^{\mathrm{e}}$ siècle $^{23}$.

\section{Les Praenotamenta : un art poétique en miniature}

Josse Bade ouvre son commentaire aux comédies de Térence par une longue introduction qu'il nomme lui-même Praenotamenta. Il s'agit de "notes préliminaires " plutôt que d'une "préface » à proprement parler. Cette section est composée de 26 chapitres qui forment un traité de poétique en miniature ${ }^{24}$ auxquels il adjoint une série de remarques préliminaires sur le prologue et la première scène de L'Andrienne. L'humaniste y développe une ample réflexion sur la comédie. Partant d'une définition de la poésie, Bade aborde ensuite l'origine de la comédie, ses caractéristiques et mène une longue réflexion sur la scénographie antique. Les deux derniers chapitres traitent de la vie et des œuvres de Térence.

Dans le premier chapitre de ses Praenotamenta, consacré à la définition du poète et à sa dignité ("Quid sit poeta et quanta eius dignitas»), Bade commence par reprendre à son compte la théorie ficinienne des quatre fureurs, dans laquelle s'intègre l'inspiration poétique. Selon lui, nul ne peut être poète s'il n'est saisi par une inspiration divine. Il développe ensuite la comparaison de l'œuvre poétique et de l'œuvre divine en six points. À partir du premier élément de comparaison, la création ex nihilo, Bade développe une défense de la poésie. L'auteur de comédies (et aussi le poète en général) 
est à l'image de Dieu puisqu'il crée des fictions, c'est-à-dire des événements qui n'ont pas existé ; il crée donc à partir du néant. Bade s'appuie sur une distinction de la rhétorique cicéronienne déjà employée par Donat dans son commentaire à L'Andrienne : Cicéron, dans le De inventione, distingue historia, fabula et argumentum ${ }^{25}$. La fabula est un récit mythologique, légendaire, comme le sont les sujets de tragédies. L'historia est un récit historique, relatant des événements ayant réellement eu lieu. Quant à l' argumentum, récit vraisemblable ou réaliste, il développe des éléments n'ayant pas existé mais conformes à la vraisemblance; le meilleur exemple en est les sujets de comédies :

Sed ut de hac re etiam aliquid dicam, scies ut innuit Cicero in primo rhetoricorum ad Herennium quod narrationum quae in negotiis sunt, triplex est materia. Aut enim historia contexitur, aut argumentum componitur, aut fabula fingitur. "Historia ", inquit, " est res gesta sed ab etatis nostre memoria remota ». " Argumentum est ficta res quae tamen fieri potuit, velut argumenta comediarum ». "Fabula est quae neque veras neque verisimiles continet res, ut he que a tragediis tradite sunt $\%$. Hec Cicero.

Ex quibus habetur quod qui scribunt, aut omnino veritatem sine aliquo figmento sequuntur, ut sunt historiographi, quos Lucanus imitatur (quapropter multi eum poetam negant, et Quintilianus oratori quam poete magis imitandum dicit), aut partim veras, partim fictas scribunt, ut poete qui cum rebus gestis fabulas admiscent, quales sunt poete heroici qui, regum et principum gesta describentes, divinas personas intermiscent. Sub quarum pretextu fingunt fabulosa et que neque vera sunt neque verisimilia, saltem ut narrantur quales sunt fabule de deorum adulteriis, de incestu Iovis, nunc in cygni, nunc in tauri, nunc in pluvie auree, nunc in alterius specie versi et de bellis a deo cum mortalibus gestis.

Mais pour dire encore quelque chose à ce sujet, il faut savoir que Cicéron affirme dans le premier livre de la Rhétorique à Herennius que la matière des narrations qu'on rencontre dans les procès, est triple. En effet, soit on tisse une histoire, soit on compose une fiction, soit on invente un récit fabuleux. « L'histoire est », dit-il, « un événement qui s'est déroulé, mais éloigné de la mémoire de notre époque ». « La fiction est un événement imaginé qui aurait pu toutefois avoir lieu, comme les sujets des comédies ». "Le récit fabuleux, comme ceux tirés des tragédies, ne contient des événements ni vrais ni vraisemblables ». Voilà ce que dit Cicéron.

Il s'ensuit que les écrivains soit suivent complètement la vérité sans recourir à aucune fiction, comme les historiens, que Lucain imite (c'est pourquoi beaucoup de gens affirment qu'il n'est pas poète et Quintilien dit qu'il constitue un modèle davantage pour l'orateur que pour le poète), soit écrivent pour partie des choses vraies et pour partie des choses fictives, comme les poètes qui mêlent des récits fabuleux aux événements qui se sont déroulés; les poètes héroïques, par exemple, tout en décrivant les actions des rois et des princes, ajoutent des personnages divins. Ces derniers leur servent d'habillage pour forger des histoires merveilleuses qui ne sont ni vraies ni vraisemblables comme, par exemple, les récits fabuleux ayant pour sujets les adultères des dieux, l'inceste de Jupiter qui prend l'apparence tantôt d'un cygne, tantôt d'un taureau, tantôt d'une pluie d'or, tantôt d'autre chose et les guerres menées par un dieu contre les hommes.

Bade proclame la noblesse de la fiction absolue. Il défend les œuvres totalement fictives, conformes ni à la vérité ni à la vraisemblance (fabula), dans le but de réfuter l'accusation de mensonge proférée contre la poésie. Les vérités contenues dans les poèmes sont cachées sous le voile de la fiction poétique (sub poetico figmento); il appartient au lecteur de déceler ces significations élevées contenues dans les fictions poétiques qui sont donc dignes, non de foi, mais d'admiration. Bade fait ainsi usage de notions rhétoriques au service de la réfutation des mensonges des poètes et développe la notion humaniste de fiction poétique. concerne les différents types de poèmes ("Quotuplicia sint poetarum scripta») : la 
distinction entre les modes d'énonciation "dramatique » (dramatica), " exégématique » ou «narratif» (exegematica) et "mixte» (mixta), empruntée à l'Ars grammatica de Diomède. Elle lui permet de réunir les genres théâtraux (tragédie, comédie et mime) dans la catégorie du dramatique, en y adjoignant le genre de l'églogue, puisque dans ces textes, le poète, sans prendre lui-même la parole, met en scène des personnages en action. La catégorie de l'« exégématique » ou «narratif » recouvre la poésie didactique, où seul le poète parle. Enfin la catégorie "mixte » mêle les deux précédentes et s'applique à l'historiographie pour la prose et à l'épopée pour la poésie. Le commentaire de Bade révèle un intérêt marqué pour la question des genres littéraires et en particulier pour une étude approfondie des deux genres théâtraux principaux que sont la tragédie et la comédie.

À partir du quatrième chapitre, Bade développe et analyse des notions proprement théâtrales, qu'il s'agisse des différences génériques entre comédie et tragédie, de l'architecture et de la scénographie du théâtre antique ou de la structure de la comédie antique. La fin des Praenotamenta revient sur la notion de decorum : les chapitres XX à XXIII portent les titres respectifs " De decoro et primo personarum ", "De rerum decoro ", «De verborum decoro » et «De decoro totius operis ». Comme l'a montré Jean Lecointe, ces chapitres sont la reprise sous forme synthétique d'« observations qu'il avait présentées de façon plus linéaire et dispersée dans le commentaire d'Horace, tout en enrichissant sa doctrine sur certains points ${ }^{26} »$. Le terme de decorum est d'ailleurs difficile à traduire : « convenance » ou « justesse » insistent sur l'idée d'adaptation, de bienséance également, mais s'y mêle une notion d'élégance quand il est question du style. S'appuyant sur une notion cicéronienne, comme nous allons le voir, Bade l'applique au théâtre et en particulier au traitement des personnages. Il place en effet en tête de ces chapitres le personarum decorum.

\section{Notions rhétoriques dans le commentaire de Bade : l'exemple du decorum}

Le commentaire lui-même des pièces de Térence recourt à cette notion de decorum. Dans son commentaire du v. 7 du prologue de L'Eunuque, Bade explique l'expression " bene uortendo" ("en traduisant bien ») qui est employée chez Térence à propos de Luscius Lanuvius, adversaire du poète. La notion de decorum est appliquée par Bade à la question de l'imitation et en particulier au problème de la traduction des pièces grecques par les auteurs comiques latins :

Potest "bene" etiam exponi pro eo quod est fideliter, id est de verbo ad verbum, non commiscendo nec commutando fabulas; quod Luscius credidit bene factum esse, cum tamen in ea re erret. Nam dicit Horatius in Arte poetica :

Nec verbo verbum curabis reddere fidus

Interpres nec desilies imitator in arctum,

Vnde pedem proferre pudor vetet, aut operis lex

id est non astringes te ad imitationem talem ut nihil immutare, commiscere aut addere audeas, ut fecit Luscius qui id bene factum putavit et ideo poete vitio dedit quod commiscuerit Menandri fabulas. Qui ergo vertendo bene non revera, sed sicut ipse existimavit, ut dictum fuit in prologo Andrie "quorum emulari exoptat negligentiam / potius quam istorum obscuram diligentiam", non que revera fuerit negligentia, sed existimata ab imperitis. Bene ergo vertendo secundum opinionem Luscii, id est fideliter, et male, id est indecenter, describendo fecit ex grecis bonis latinas non bonas. Quod primo illi dat vitio, deinde quia in Phasmate Menandri non servavit forte decorum. 
On peut même expliquer «bien " par "fidèlement ", c'est-à-dire mot à mot, sans mêler ni changer les pièces. C'est ce que Luscius croit avoir bien fait, alors qu'il se trompe à ce sujet. Car Horace dit dans son Art poétique [v. 133-135] :

En traducteur fidèle, tu ne t'appliqueras pas à rendre

Mot pour mot, et en imitant, tu ne t'engageras pas dans une voie trop étroite,

De laquelle la timidité ou la loi du genre t'empêcherait de sortir.

C'est-à-dire tu ne t'attacheras pas à une imitation qui fasse que tu n'oses rien changer, mêler ou ajouter, comme l'a fait Luscius qui a cru bien faire ainsi et a reproché au poète d'avoir mêlé plusieurs pièces de Ménandre. Donc « en traduisant bien ", c'est-à-dire non au pied de la lettre, mais selon son propre sentiment, comme cela a été dit dans le prologue de L'Andrienne [v. 20-21] : « ces auteurs dont il désire imiter la négligence plutôt que l'obscur soin de ces autres ». Car ce n'est pas en réalité de la négligence, mais c'est ainsi que la jugent les ignorants. Donc «en traduisant bien ", selon l'opinion de Luscius, c'est-à-dire fidèlement, et en transposant mal, c'est-à-dire d'une manière qui ne convient pas, à partir de bons textes grecs, il a fait de mauvais textes latins. Voilà ce qu'il lui reproche d'abord, ensuite de n'avoir pas gardé ce qui convient dans le Phasma de Ménandre.

Dans ce commentaire de forme lemmatique, où il reprend à plusieurs reprises les deux mots de Térence qu'il commente (« bene uortendo »), Bade se rattache à une conception humaniste de l'imitation qui est devenue traditionnelle après Pétrarque et Politien : il revendique la liberté de création de l'imitateur qui ne s'assujettit pas à ses modèles mais en fait une réécriture personnelle. Il recourt non seulement au terme decorum, mais aussi à l'adverbe indecenter de la même famille, mots qu'il associe au processus de l'imitation (imitationem).

Dans l'Antiquité classique, cette notion de decorum, traduction du grec $\pi \rho \varepsilon ́ \pi o v$ [prepon] comme l'a montré Jean Lecointe ${ }^{27}$, est développée par Cicéron tant du point de vue rhétorique que du point de vue éthique dans deux textes (Orator, 71; De Officiis, I, 93) que Bade connaît assurément. Le second, quoique de nature philosophique, rappelle que le respect du decorum (decorum seruare) fait partie des devoirs du poète et l'applique de manière tout à fait intéressante aux personnages : il convient au poète d'écrire «ce qui est digne de chaque personnage» («id quod quaque persona dignum est»). Ainsi, comme le souligne Jean Lecointe, « le decorum de la représentation poétique des personnages s'y trouve mis explicitement en rapport avec le decorum de l'action humaine réelle, envisagée d'un point de vue éthique ${ }^{28} »$.

Bade est particulièrement attentif au decorum des personnages et le développe de manière originale en s'intéressant aux sentiments (affectiones ${ }^{29}$ ). En effet, selon lui, le poète ne doit pas se limiter à prendre en compte l'âge, le sexe et la condition de ses personnages, mais aussi leurs sentiments. Son commentaire intègre dès lors de nombreuses notations psychologiques sur les personnages térentiens :

Maxime autem omnium animi affectiones ad decorum faciunt. Nam non satis est considerare aetatem sexum, patriam, statum et fortunam, nisi animus eius qui loquitur consideretur. Aliter enim loquitur rex in extremis aduersitatibus depressus: aliter in prosperitate elatus. [...] Quapropter boni poetae doctique viri quum faciunt aliquas personas loquentes dant ipsis talem sermonem qualem reuera haberent si ipsi in propria persona loquerentur.

Plus que toute chose les affections de l'âme ont trait au decorum. Car il ne suffit pas de prendre en considération l'âge, le sexe, la condition et la fortune si l'on ne considère pas l'âme de celui qui parle. En effet, un roi parle d'une manière quand il est accablé par des malheurs extrêmes et d'une autre quand il est porté par une situation prospère. [...] C'est pourquoi les bons poètes et les hommes savants, quand ils font parler certains personnages, leur attribuent le discours qu'eux-mêmes tiendraient réellement s'ils étaient à la place de leur personnage. 
Pour reprendre l'analyse de Jean Lecointe, « on retrouve les consignes du commentaire relatives à l'identification affective du poète avec le personnage, intégrées cette fois à une division spécifique du decorum personarum, celle des affectiones. Le caractère fortement mimétique et émotionnel du decorum humaniste n'en est que plus vivement souligné ${ }^{30} »$.

\section{Conclusion}

Le commentaire de Bade aux comédies de Térence est en définitive assez différent des commentaires des humanistes italiens, préoccupés par les explications philologiques. Comme le souligne Paul White, il n'a pas d'identité à proprement parler ${ }^{31}$. Car Josse Bade, s'il se rattache au milieu humaniste par certains traits, est avant tout un imprimeur. C'est que son commentaire n'est pas destiné à un même public que celui des humanistes italiens. Même s'il entretient des liens avec son activité d'enseignement, il ne s'inscrit nullement dans une carrière universitaire ou courtisane. Il possède avant tout une vocation de vulgarisation. Comme le note Louise Katz, "si Bade dote ses éditions d'un appareil paratextuel particulièrement développé, c'est pour tenter de satisfaire aux exigences et aux attentes du lectorat le plus large possible. En faisant la promesse de mettre à la portée de tous la lecture des chefs-d'œuvre de la littérature antique, il espérait bien faire des lecteurs ordinairement laissés pour compte des bonnes lettres les acheteurs de ses éditions ${ }^{32}$ ». En outre, le projet de Bade est aussi foncièrement moral: il revendique un usage éthique de la littérature. Les textes de Térence et leur analyse s'y prêtent tout particulièrement, comme en témoignent les différentes lectures du poète latin au fil des siècles. Le Térence de 1502 s'insère donc pleinement dans ce double projet d'imprimeur : offrir au plus grand nombre des textes de qualité et proposer une lecture morale des textes classiques latins de l'Antiquité païenne.

\section{NOTES}

1. H. W. Lawton, Térence en France au XVI $I^{e}$ siècle, Genève, Slatkine repr., 1970-1972 (1 ${ }^{\text {ère }}$ édition : Paris, Jouve, 1926), 2 vol., vol. 2, p. 9.

2. Giovanni Calfurnio, humaniste $d u \mathrm{Xv}^{\mathrm{e}}$ siècle originaire de Bergame, écrivit un commentaire à l' Heautontimoroumenos pour compléter le commentaire de Donat. Les éditions du $\mathrm{xvI}^{\mathrm{e}}$ siècle l'insèrent très souvent dans le commentaire de Donat.

3. Voir A. Poliziano, La commedia antica e l'Andria di Terenzio, éd. R. Lattanzi Roselli, Florence, Sansoni, 1973.

4. Guidonis Iuvenalis natione Cenomani in Terentium familiarissima interpretatio cum figuris unicuique scaenae praepositis, Lyon, Jean Trechsel, 1493 (voir H. W. Lawton, op. cit., vol. 1, p. 91). Comme nous le verrons plus loin, la première édition du commentaire de Bade proprement dit au texte de Térence paraît à Lyon en 1502 : P. Terentii aphri comicorum elegantissimi comedie a Guidone Iuvenale viro perquam litterato familiariter explanate: et ab Jodoco Badio Ascensio vna cum explanationibus rursum annotate atque recognite: cumque eiusdem Ascensii praenotamentis atque annotamentis suis locis 
adhibitis quam accuratissime se venundant impresse, Lyon, François Fradin, 1502 (voir H. W. Lawton, op. cit., vol.1, p.104-105). Nous avons consulté et citons, dans l'exemplaire de la Bibliothèque Nationale de France, l'édition de 1504 qui reproduit la précédente : P. Terentii Aphri, comicorum elegantissimi, Comedie a Guidone Juvenale [...] familiariter explanate, et ab Jodoco Badio Ascensio una cum explanationibus rursum annotate atque recognite, cumque ejusdem Ascensii prenotamentis atque annotamentis, Londres et Paris, Josse Bade et alii, 1504 (voir H.W. Lawton, op.cit., vol.1, p. 107-108). Les traductions, sauf indication contraire, sont nôtres.

5. Voir à ce sujet l'article de Bruno Bureau dans ce numéro.

6. P. White, Jodocus Badius Ascensius. Commentary, Commerce and Print in the Renaissance, Oxford University Press, 2013, p. 30 : "both printer and scholar». Pour une mise au point biographique récente sur Josse Bade, outre le premier chapitre de l'ouvrage de Paul White (ibid., p. 1-33), voir l'introduction de la thèse de Louise Katz, soutenue en 2013 à l'École pratique des Hautes Études : L. Katz, La Presse et les lettres. Les épîtres paratextuelles et le projet éditorial de l'imprimeur Josse Bade (c. 1462-1535), dir. P. Galand, EPHE, décembre 2013, p. 1-6 (fichier PDF aimablement fourni par l'auteur). Pour une biographie détaillée, mais plus ancienne, voir Ph. Renouard, Bibliographie des impressions et des ceuvres de Josse Bade Ascensius, Paris, E. Paul et fils et Guillemin, 1908, vol.1, p. 1-38.

7. L. Katz, op. cit., p. 393.

8. P. White, op. cit., p. 32.

9. L. Katz, op. cit., p. 13.

10. Cette édition est consultable sur gallica.bnf.fr.

11. Sur Guy Jouenneaux, voir J.-B. Hauréau, Histoire littéraire du Maine, Paris, 1870-1877 (repr. Genève, Slatkine, 1969), vol. 6, p. 186-191. Jouenneaux est également l'auteur d'un commentaire aux Elegantiae linguae latinae de Lorenzo Valla qui paraît pour la première fois en 1496 et que Bade fait sortir de ses propres presses en 1508.

12. L. Katz, op. cit., p. 6, 77. Le texte de l'épître est donné en annexe de la thèse, p. 428.

13. P. White, op. cit., p. 216 sq.

14. Ibid., p. 219 : «These rules illustrate the uses to which Terence's text might be put in the schoolroom: not only as a source of sententiae but a pretext for the delivery of moral lessons quite far removed from what is said in the text itself»; "Clearly the commentary text here directly reflects Badius's practice as a schoolmaster: he would have dictated numbered lists of this type to be copied down by students ".

15. M. Lebel, Les Préfaces de Josse Bade (1462-1535) humaniste, éditeur-imprimeur et préfacier, Louvain, Peeters, 1988, p. 152-154.

16. L. Annei Senecae Tragoediae, pristinae integritati restituae per exactissimi judicii viros, post Avantium et Philologum : D. Erasmum Roterodamum, Gerardum Vercellanum, Aegidium Maserium, cum metrorum praesertim tragicorum ratione ad calcem operis posita. Explanatae [...] tribus commentariis : G. Bernardino Marmita, [...] Daniele Gaietano, [...] Jodoco Badio Ascensio, Paris, Josse Bade, 1514.

17. Ph. Renouard, op. cit., vol. 3, p. 252.

18. M. Lebel, op. cit., p. 152 (la traduction est de M. Lebel).

19. Voir note 4.

20. Voir dans ce numéro l'introduction de l'article de Bruno Bureau sur le commentaire de Donat.

21. P. White, op. cit., p. 220 : «trial run ».

22. Voir note 4 .

23. P. White, op. cit., p. 208. Harold W. Lawton mentionne dans sa liste les reprises de cette édition : voir H. W. Lawton, op. cit., vol. 1, p. 105 sq. (passim).

24. Voir Poétiques de la Renaissance. Le modèle italien, le modèle franco-bourguignon et leur héritage en France au XVI esiècle, dir. P. Galand-Hallyn et F. Hallyn, Genève, Droz, 2001, p. 261 : Perrine Galand qualifie ce texte de « véritable art poétique des débuts de la Renaissance française ». 
25. Cicéron, De inuentione, I, 27. Voir aussi Ad Herennium, I, 13. Sur la question, voir Anne Duprat, Vraisemblances. Poétique et théorie de la fiction en France et en Italie (XVI ${ }^{e}-\mathrm{XVII}^{e}$ siècles), Paris, Champion, 2009.

26. J. Lecointe, «Josse Bade et l'invention du decorum horatien », Camenae, 13 (octobre 2012), p. 6 : http://saprat.ephe.sorbonne.fr/media/a479f7ffb0c7a4aeb9b186c883744f15/camenae-13-9-

lecointe.pdf.

27. Ibid., p. 5.

28. Ibid.

29. Ibid., p. 8-9.

30. Ibid., p. 9. La liste de Bade rappelle celle des lieux de la personne (qui sont chez Quintilien, Institution oratoire, $\mathrm{V}, 10,25-27$, dans cet ordre : sexus, aetas, fortuna, condicio, animi natura).

31. P. White, op. cit., p. 226 : «In a sense Badius's commentaries have no real identity of their own: unlike the contemporary Italian humanist writers of philological and allegorical commentaries, Badius was more interested in reaching as wide a readership as possible than in enhancing his own scholarly reputation "; " the success they achieved was due to their general appeal ».

32. L. Katz, op. cit., p. 328.

\section{AUTEUR}

\section{LAURE HERMAND-SCHEBAT}

Université Jean Moulin-Lyon 3, UMR 5189 (HiSoMA) 